\title{
Conditions for Triangular Decoupling Control
}

\author{
Hung T. Nguyen *, and Steven W. Su *
}

The main purpose of this paper is to explore the relationship of two existing conditions for the triangular decoupling problem. The first one is the TriangularDiagonal-Dominance (TDD) condition proposed by Hung and Anderson. The second one is the stable coprime factorization described condition proposed by Gomez and Goodwin in 2000, which has been proved as a necessary and sufficient condition for the triangular decoupling problem. This paper proves that the two conditions are actually equivalent. It also provides easy-to-use criteria for the assessment of the solvability of the triangular decoupling problem.

Index Terms - Linear multivariable systems, Triangular decoupling, TriangularDiagonal-Dominance, Stable coprime factorization.

\section{Introduction}

The research of decoupling of multivariable systems has attracted great attention (Hung and Anderson(1979); Anderson and Hung(1978); Kouvaritakis(1996); Gomez and Goodwin(2000)). Since diagonal decoupling has certain fundamental design limitations, there has also been interest in triangular decoupling. Earlier work on triangular decoupling set the multivariable design problem on the framework of finding canonical forms of matrices over Euclidean domains. In this framework, Hung (now Hung T. Nguyen, an author of this paper) and Anderson (Anderson and Hung(1978)) (Hung and Anderson(5-7, June, 1979)) (Hung and Anderson(1979)) proposed efficient triangularisation approaches for a class of multivariable systems which satisfy the Triangular-Diagonal-Dominance (TDD) condition. Recently, several new efficient methods, such as stable coprime factorisation based approach (Gomez and Goodwin(2000)) and static feed back approach (Kouvaritakis(1996)) (Kouvaritakis and Skarpetis(2000)) have been adopted to explore the triangular decoupling problem. In (Gomez and Goodwin(2000)), Gomez and Goodwin systematically examined the problem of decoupling design based on an algebraic approach and the use of coprime factorisation over the ring of proper and stable rational functions (Vidyasagar(1985)), and proposed a necessary and sufficient condition for the solvability of the triangular decoupling problem (see Problem 1 below). The main purpose of this paper is to investigate the relationship between TDD condition proposed in (Hung and Anderson(1979)) and the stable coprime factorisation described condition (Theorem 1 of (Gomez and Goodwin(2000))) provided by Gomez and Goodwin.

Gomez and Goodwin proposed the following definition of the triangular decoupling problem.

\footnotetext{
*Hung T. Nguyen and Steven W. Su are with Faculty of Engineering, University of Technology, Sydney (UTS), P.O. Box 123, Broadway NSW 2007, Australia. Email: $\{$ Hung.Nguyen $\}\{$ Steven.Su\}@uts.edu.au.
} 


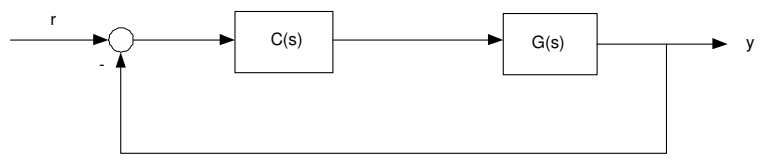

Figure 1.. A closed loop system.

Problem 1 Consider a controlled process $G(s)$ as shown in Figure 1, which is described by a $m \times m$, full-rank, proper rational matrix. The triangular decoupling problem is the design of a controller $C(s)$ for the plant $G(s)$ to make the closed loop complementary sensitivity function $T(s)$ and the sensitivity function $S(s)$ lower triangular, and to guarantee the internal stability of the closed loop.

Hung and Anderson (Hung and Anderson(1979)) showed that if $G(s)$ can be precompensated to TDD (see Definition 1) the triangular decoupling problem is solvable. (Hung and Anderson(1979)) also provided approaches to reduce multivariable design problem to scalar design problems. In (Gomez and Goodwin(2000)), Gomez and Goodwin provided a necessary and sufficient condition for Problem 1 based on stable coprime factorisation (Theorem 1 of (Gomez and Goodwin(2000))).

This paper will prove the equivalence of the stable coprime factorisation condition and TDD condition so that some easy-to-use rules developed in (Hung and Anderson(1979)) can be applied to evaluate the solvability of the triangular decoupling problem even without necessarily performing triangularisation. This paper also provides an MFDs described method for readers to assess the solvability of the triangular decoupling problem as an alternative to stable coprime factorization approach.

This paper is organized as follows. All main results are presented in section 2 . The condition provided by Gomez and Goodwin is given in Theorem 1. Theorem 2 proves the TDD condition is equivalent to an MFDs described condition. The main result is given in Theorem 3 which proves the TDD condition is actually equivalent to the condition given by Gomez and Goodwin. Lemma 3 proposed a sufficient condition for both lower and upper triangular decoupling. In Section 3, two illustrative examples are presented to explain and justify the results.

\section{TDD and stable coprime factorisation based condition}

In order to clarify our discussion, the following symbols and notations are adopted from literature (Gomez and Goodwin(2000)) (Vidyasagar(1985)) (Hung and Anderson(1979)):

- $\mathcal{R}[s]$ : the set of polynomials in the indeterminate $s$ with coefficients in the field $\mathcal{R}$ of real numbers.

- $\mathcal{R}(s)$ : the field of fractions associated with $\mathcal{R}[s]$ (or the set of all possible transfer functions of scalar, lumped, linear time invariant systems).

- $\mathcal{M}(\mathcal{R})$ : the set of matrices with elements in $\mathcal{R}[s]$. 
- $\mathcal{M}(\mathcal{F})$ : the set of matrices with elements in $\mathcal{R}(s)$.

- $\mathcal{S}$ : the subset of $\mathcal{R}(s)$ consisting of all rational functions that are bounded at infinity, and whose poles all have negative real parts.

- $\mathcal{U}$ : the set of units of $\mathcal{S}$, i.e. functions in $\mathcal{S}$ whose reciprocal also belongs to $\mathcal{S}$.

- $\mathcal{M}(\mathcal{S})$ : the set of matrices with elements in $\mathcal{S}$.

- $\mathcal{U}_{\mathcal{M}}(\mathcal{S})$ : the set of unimodular matrices of $\mathcal{M}(\mathcal{S})$.

- $\mathcal{C}_{+}$: the closed right half plane.

- $\mathcal{C}_{+e}$ : the extended right half plane, i.e. $\mathcal{C}_{+}$together with the point at infinity.

- $X_{+}(s)$ : the sum of those strictly proper terms in a partial fraction expansion of $X(s) \in \mathcal{M}(\mathcal{F})$ with poles in $\mathcal{C}_{+}$.

- $X_{-}$: the sum of those strictly proper terms in a partial fraction expansion of $X(s) \in \mathcal{M}(\mathcal{F})$ with poles in the (open) left half plane.

- $|X(s)|$ : the determination of $\mathrm{X}(\mathrm{s})$. That is $|X(s)|=\operatorname{det}(X(s))$

- $\delta[X(s)]$ : the McMillan degree of a proper transfer function matrix $X(s) \in$ $\mathcal{M}(\mathcal{F})$. It is not hard to prove that $\delta[X(s)]=\delta\left[X_{+}\right]+\delta\left[X_{-}\right]$.

- $\tilde{X}(s)$ : the diagonal part of the square matrix $X(s)$. That is $\tilde{X}(s)=$ $\operatorname{diag}\left[x_{11}(s), \cdots, x_{m m}(s)\right]$.

Definition 1 (Hung and Anderson(1979)) For a proper rational matrix $G(s) \in$ $\mathcal{R}(s)^{m \times m}$, assume there exist a unimodular matrix $U(s) \in \mathcal{U}_{\mathcal{M}}(\mathcal{S})$ such that

$$
P(s)=G(s) U(s),
$$

where $P(s) \in \mathcal{R}(s)^{m \times m}$ is a lower triangular matrix.

If

$$
\delta\left[P_{+}(s)\right]=\delta\left[\tilde{P}_{+}(s)\right] .
$$

then, we say $P(s)$ is triangular-diagonal-dominant (TDD) and $G(s)$ can be precompensated to TDD.

The following notations are cited from (Gomez and Goodwin(2000)) to highlight some important issues about stable coprime factorization:

Assume a stable coprime factorization of a full-rank, proper rational matrix $G(s) \in \mathcal{R}(s)^{m \times m}$ can be expressed in the following from:

$$
G(s)=N_{p} \cdot D_{p}^{-1}
$$

where, $N_{p} \in \mathcal{S}^{m \times m}$ and $D_{p} \in \mathcal{S}^{m \times m}$ are stable transfer function matrices and right coprime. Suppose that

$$
N_{p} U_{N_{p}}=L_{N_{p}}
$$

and

$$
\bar{D}_{p}=D_{p} U_{N_{p}}
$$


where $U_{N_{p}} \in \mathcal{U}_{\mathcal{M}}(\mathcal{S})$ (an unimodular matrix of $\mathcal{M}(\mathcal{S})$ ), and $L_{N_{p}}$ is lower triangular. Then,

$$
G(s)=L_{N_{p}} U_{N_{p}}^{-1} D_{p}^{-1}=L_{N_{p}}\left(D_{p} U_{N_{p}}\right)^{-1}=L_{N_{p}} \bar{D}_{p}^{-1} .
$$

Further assume

$$
\bar{U}_{D_{p}} \bar{D}_{p}=\bar{L}_{D_{p}}
$$

where, $\bar{U}_{D_{p}} \in \mathcal{U}_{\mathcal{M}}(\mathcal{S})$, and $\bar{L}_{D_{p}}$ is lower triangular. Then,

$$
G(s)=L_{N_{p}}\left(\bar{U}_{D_{p}}^{-1} \bar{L}_{D_{p}}\right)^{-1}=\left(L_{N_{p}} \bar{L}_{D_{p}}^{-1}\right) \bar{U}_{D_{p}}=P(s) \bar{U}_{D_{p}}
$$

where $P(s)=G(s) \bar{U}_{D_{p}}^{-1}$ is a lower triangular transfer function matrix.

As claimed in (Gomez and Goodwin(2000)) $L_{N_{p}}$ and $\bar{L}_{D_{p}}$ are invariants up to postmultiplication by a lower triangular unimodular matrix of $\mathcal{M}(\mathcal{S})$ and are independent of particular factorisation of $G(s)$. Therefore, the non-minimum phase zeros of $\bar{L}_{D_{p}} \in \mathcal{M}(\mathcal{S})$ (including multiplicities) and the zeros at infinity of each diagonal entry are invariant.

Gomez et al proposed a necessary and sufficient condition for Problem 1 based on the stable coprime factorised form (2.3).

Theorem 1 (Gomez and Goodwin(2000)) For a full-rank, proper rational matrix $G(s) \in \mathcal{R}(s)^{m \times m}$, the following two statements are equivalent:

i) the triangular decoupling problem (Problem 1) is solvable for $G(s)$;

ii) each diagonal entry of $L_{N_{p}}$ is coprime with the corresponding diagonal entry of $\bar{L}_{D_{p}}\left(L_{N_{p}}\right.$ and $\bar{L}_{D_{p}}$ are as defined in equation (2.3)).

Proof: See Theorem 1 of (Gomez and Goodwin(2000)).

Lemma 1 (Hung and Anderson(1979)) For a lower triangular proper transfer function matrix $P(s) \in \mathcal{R}(s)^{m \times m}$, there exist right matrix fraction descriptions (MFDs):

$$
P(s)=N_{L}(s) D_{L}^{-1}(s),
$$

where $N_{L}(s)$ and $D_{L}(s)$ are lower triangular polynomial matrices.

Proof: See Lemma 2 of (Hung and Anderson(1979)).

Theorem 2 For a proper rational matrix $G(s) \in \mathcal{R}(s)^{m \times m}$, assume there exist a unimodular matrix $U \in \mathcal{U}_{\mathcal{M}}(\mathcal{S})$ such that

$$
P(s)=G(s) U(s),
$$

where $P(s) \in \mathcal{R}(s)^{m \times m}$ is a lower triangular matrix.

Suppose $P(s)$ has the following MFDs form:

$$
P(s)=N_{L}(s) D_{L}^{-1}(s),
$$

where $N_{L}(s) \in \mathcal{R}[s]^{m \times m}$ and $D_{L}(s) \in \mathcal{R}[s]^{m \times m}$ are lower triangular polynomial matrices and right coprime. Then, the following two statements are equivalent: 
i) Each diagonal entries of $N_{L}(s)$ and $D_{L}(s)$ have no common non-minimal phase zeros.

ii) $P(s)$ is $T D D$ (i.e. $G(s)$ can be precompensated to TDD).

Proof: For a square polynomial matrix $D(s) \in \mathcal{M}(\mathcal{R})$ we denote

$$
\mathcal{N}_{\mathcal{R}}^{+}(D(s))
$$

as the number of non-minimal phase zeros (including multiplicity) of $|D(s)|$.

Consider that $N_{L}(s)$ and $D_{L}(s)$ in equation (2.5) are right coprime we have

$$
\delta\left[P_{+}(s)\right]=\mathcal{N}_{\mathcal{R}}^{+}\left(D_{L}(s)\right) .
$$

From equation (2.5), we can get

$$
\tilde{P}(s)=\tilde{N}_{L}(s) \tilde{D}_{L}^{-1}(s) .
$$

Now, if condition i) is satisfied, i.e. each diagonal entries of $N_{L}(s)$ and $D_{L}(s)$ have no common non-minimal phase zeros, then $\tilde{N}_{L}(s)$ and $\tilde{D}_{L}(s)$ are coprime over the closed right half plane $\mathcal{C}_{+}$. Then, we have

$$
\delta\left[\tilde{P}_{+}(s)\right]=\mathcal{N}_{\mathcal{R}}^{+}\left(\tilde{D}_{L}(s)\right) .
$$

Consider that $D_{L}(s)$ is lower triangular matrix, we can see that

$$
\left|\tilde{D}_{L}(s)\right|=\left|D_{L}(s)\right| .
$$

Thus,

$$
\mathcal{N}_{\mathcal{R}}^{+}\left(\tilde{D}_{L}(s)\right)=\mathcal{N}_{\mathcal{R}}^{+}\left(D_{L}(s)\right)
$$

Based on equations (2.6), (2.7) and (2.8), we conclude that

$$
\delta\left[\tilde{P}_{+}(s)\right]=\delta\left[P_{+}(s)\right] .
$$

Thus $P(s)$ is TDD

On the other hand if i) is incorrect, i.e. some diagonal entries of $N_{L}(s)$ and $D_{L}(s)$ have common non-minimal phase zeros, then $\tilde{N}_{L}(s)$ and $\tilde{D}_{L}(s)$ are not coprime over the closed right half plane $\mathcal{C}_{+}$. Thus,

$$
\delta\left[\tilde{P}_{+}(s)\right]<\mathcal{N}_{\mathcal{R}}^{+}\left(\tilde{D}_{L}(s)\right)=\mathcal{N}_{\mathcal{R}}^{+}\left(D_{L}(s)\right) .
$$

Based on equations (2.6) and (2.9), we have

$$
\delta\left[\tilde{P}_{+}(s)\right]<\delta\left[P_{+}(s)\right] .
$$

Then, ii) is also incorrect.

Lemma 2 Consider a lower triangular proper transfer function matrix $P(s) \in$ $\mathcal{R}(s)^{m \times m}$. Assume $P(s)$ can be expressed in a stable coprime factorized form:

$$
P(s)=L_{N_{p}} \bar{L}_{D_{p}}^{-1} .
$$

Where, $L_{N_{p}}$ and $\bar{L}_{D_{p}}$ are stable lower triangular matrices and coprime each other.

Then, there is no infinity zero in each diagonal entry of $\bar{L}_{D_{p}}$. 
Proof: See Appendix A.

Now, we prove the equivalence of the stable coprime described condition and TDD condition.

Theorem 3 (Main result) For a proper rational matrix $G(s) \in \mathcal{R}(s)^{m \times m}$, assume there exist a unimodular matrix $U(s) \in \mathcal{U}_{\mathcal{M}}(\mathcal{S})$ such that

$$
P(s)=G(s) U(s)
$$

where $P(s) \in \mathcal{R}(s)^{m \times m}$ is a lower triangular matrix.

Suppose $P(s)$ can be expressed in a stable coprime factorized form:

$$
P(s)=L_{N_{p}} \bar{L}_{D_{p}}^{-1} \text {. }
$$

Where, $L_{N_{p}} \in \mathcal{R}(s)^{m \times m}$ and $\bar{L}_{D_{p}} \in \mathcal{R}(s)^{m \times m}$ are stable lower triangular matrices and coprime each other (See equation (2.3)). Then, the following two statements are equivalent:

i) each diagonal entry of $L_{N_{p}}$ is coprime with the corresponding diagonal entry of $\bar{L}_{D_{p}}$.

ii) $P(s)$ is $T D D$ (i.e. $G(s)$ can be precompensated to TDD).

Proof: As

$$
P(s)=G(s) \bar{U}(s)=L_{N_{p}} \bar{L}_{D_{p}}^{-1},
$$

we have $\delta\left[P_{+}(s)\right]$ equals to the number of non-minimal phase zeros of $\bar{L}_{D_{p}}$.

As $\bar{L}_{D_{p}}$ is a lower triangular matrix, all its zeros contain in its diagonal entries. If we define

$$
\mathcal{N}_{\mathcal{F}}^{+}\{a(s) / b(s)\}
$$

as the number of remaining non-minimal phase zeros (including multiplicity) of $a(s) \in \mathcal{R}(s)$ after possible cancellation with the zeros (including multiplicity) of $b(s) \in \mathcal{R}(s)$, where $a(s)$ and $b(s)$ are two scalar transfer functions. Then, we have $\delta\left[\tilde{P}_{+}(s)\right]=\sum_{i=1}^{m} \mathcal{N}_{\mathcal{F}}^{+}\left\{l_{N_{p}}(s)_{i i} / \bar{l}_{D_{p}}(s)_{i i}\right\}$, where $l_{N_{p}}(s)_{i i}$ and $\bar{l}_{D_{p}}(s)_{i i}$ are the $i_{t h}$ diagonal entries of $L_{N_{p}}$ and $\bar{L}_{D_{p}}$ respectively.

Now, if $P(s)$ is TDD, then we have

$$
\delta\left[P_{+}(s)\right]=\delta\left[\tilde{P}_{+}(s)\right] .
$$

That is the number of non-minimal phase zeros of $\bar{L}_{D_{p}}$ equals to

$$
\delta\left[\tilde{P}_{+}(s)\right]=\Sigma_{i=1}^{m} \mathcal{N}_{\mathcal{F}}^{+}\left\{l_{N_{p}}(s)_{i i} / \bar{l}_{D_{p}}(s)_{i i}\right\}
$$

This means that there are no common non-minimal phase zeros between each diagonal entry of $L_{N_{p}}$ and $\bar{L}_{D_{p}}$. Furthermore, according to Lemma $2, \bar{l}_{D_{p}}(s)_{i i}$ has no zeros at infinity. Thus, we have each diagonal entry of $L_{N_{p}}$ is coprime with the corresponding diagonal entry of $\bar{L}_{D_{p}}$.

On the other hand, if each diagonal entry of $L_{N_{p}}$ is coprime with the corresponding diagonal entry of $\bar{L}_{D_{p}}$, then there are no common non-minimal phase zeros between each diagonal entry of $L_{N_{p}}$ and $\bar{L}_{D_{p}}$. Thus, the number of nonminimal phase zeros of $\bar{L}_{D_{p}}$ equals to $\delta\left[\tilde{P}_{+}(s)\right]=\sum_{i=1}^{m} \mathcal{N}_{\mathcal{F}}^{+}\left\{l_{N_{p}}(s)_{i i} / \bar{l}_{D_{p}}(s)_{i i}\right\}$. This ensure that $P(s)$ is TDD. 
Remark 1 Readers may find it helpful to compare the statement and proof of Theorem 2 and 3. The two theorems are quite similar. The main difference is Theorem 2 ( equation (2.5)) is stated in the Euclidean domain $\mathcal{R}[s]$, while Theorem 3 ( equation (2.11) ) is in another Euclidean domain $\mathcal{R}(s)$.

Theorems 1-3 imply that the TDD condition, stable coprime factorization based condition, and MFDs based condition are all equivalent. They are all necessary and sufficient conditions for triangular decoupling problem. In (Hung and Anderson(1979)), some easy-to-use criteria was developed to verify TDD property. These criteria can also be applied to determine the solvability of decoupling problem. For example, the following lemma as well as its proof are mainly from Lemma 6 at Page 457 of (Hung and Anderson(1979)). In some cases, this lemma can determine the solvability of both lower and upper triangular decoupling problem without performing triangularization procedure (see Example $1)$.

Lemma 3 Consider a $m \times m$, full-rank, proper rational matrix $G(s)$. If

$$
\delta\left[|G(s)|_{+}\right]=\delta\left[G_{+}(s)\right],
$$

then both lower triangular decoupling problem and upper triangular decoupling problem is solvable for $G(s)$

Proof: Assume $P(s)=G(s) U(s)$ where $U(s) \in \mathcal{U}_{\mathcal{M}}(\mathcal{S})$ is a unimodular and $P(s)$ is in either upper or lower triangular form. Then it is easy to see

$$
\delta\left[|G(s)|_{+}\right]=\delta\left[|P(s)|_{+}\right],
$$

and

$$
\delta\left[G_{+}(s)\right]=\delta\left[P_{+}(s)\right]
$$

Considering Equation (2.14), we have

$$
\delta\left[|P(s)|_{+}\right]=\delta\left[P_{+}(s)\right] .
$$

This proves $P(s)$ is TDD.

\section{Example}

The following two examples are from (Hung and Anderson(1979)) and (Gomez and Goodwin(2000)) respectively.

Example 1 Consider the following transfer function matrix (Hung and Anderson(1979))

$$
G(s)=\left[\begin{array}{ll}
\frac{1}{s+1} & \frac{1}{s-2} \\
\frac{1}{s+1} & \frac{1}{s+1}
\end{array}\right] .
$$

It can be transferred to a lower triangular form:

$$
\begin{aligned}
P(s) & =G(s) U(s) \\
& =\left[\begin{array}{ll}
\frac{1}{s+1} & \frac{1}{s-2} \\
\frac{1}{s+1} & \frac{1}{s+1}
\end{array}\right]\left[\begin{array}{cc}
0 & 1 \\
1 & -\frac{s-2}{s+1}
\end{array}\right] \\
& =\left[\begin{array}{ll}
\frac{1}{s-2} & 0 \\
\frac{1}{s+1} & \frac{3}{(s+1)^{2}}
\end{array}\right] .
\end{aligned}
$$


The MFDs form and stable coprime factorization form of $P(s)$ are shown as follows:

$$
\begin{aligned}
P(s) & =\left[\begin{array}{ll}
\frac{1}{s-2} & 0 \\
\frac{1}{s+1} & \frac{3}{(s+1)^{2}}
\end{array}\right] \\
& =\left[\begin{array}{cc}
1 & 0 \\
1 & -3
\end{array}\right]\left[\begin{array}{cc}
s-2 & 0 \\
s+1 & -(s+1)^{2}
\end{array}\right]^{-1} \\
& =\left[\begin{array}{cc}
\frac{1}{s+1} & 0 \\
\frac{s-2}{(s+1)^{2}} & \frac{3}{(s+1)^{2}}
\end{array}\right]\left[\begin{array}{cc}
\frac{s-2}{s+1} & 0 \\
0 & 1
\end{array}\right]^{-1}
\end{aligned}
$$

Similarly, the upper triangular form of $G(s)$ can be calculated:

$$
\begin{aligned}
P_{u p}(s) & =G(s) U_{u p}(s) \\
& =\left[\begin{array}{cc}
\frac{1}{s+1} & \frac{1}{s-2} \\
\frac{1}{s+1} & \frac{1}{s+1}
\end{array}\right]\left[\begin{array}{cc}
1 & 0 \\
-1 & 1
\end{array}\right] \\
& =\left[\begin{array}{cc}
\frac{1}{(s-2)(s+1)} & \frac{1}{s-2} \\
0 & \frac{1}{s+1}
\end{array}\right] .
\end{aligned}
$$

The MFDs form and stable coprime factorization form of $P_{u p}(s)$ are shown as follows:

$$
\begin{aligned}
P_{u p}(s) & =\left[\begin{array}{cc}
\frac{-3}{(s-2)(s+1)} & \frac{1}{s-2} \\
0 & \frac{1}{s+1}
\end{array}\right] \\
& =\left[\begin{array}{cc}
-3 & 1 \\
0 & 1
\end{array}\right]\left[\begin{array}{cc}
(s-2)(s+1) & s+1 \\
0 & s+1
\end{array}\right]^{-1} \\
& =\left[\begin{array}{cc}
\frac{-3}{(s+1)^{2}} & \frac{1}{s+1} \\
0 & \frac{1}{s+1}
\end{array}\right]\left[\begin{array}{cc}
\frac{s-2}{s+1} & -\frac{s-2}{s+1} \\
0 & 1
\end{array}\right]^{-1} .
\end{aligned}
$$

By checking the TDD condition, the stable coprime factorisation condition (Gomez and Goodwin(2000)) and MFDs descried condition, we can see that both upper and lower triangular decoupling are solvable.

It is also easy to see that (see page 457 of (Hung and Anderson(1979)))

$$
\left(\delta\left[|G(s)|_{+}\right]=1\right)=\left(\delta\left[G_{+}(s)\right]=1\right) .
$$

Based on Lemma 3 and equation (3.1), we can easily conclude that both lower and upper triangular decoupling is achievable without necessarily transferring $G(s)$ to its upper and triangular form and calculating its stable coprime factorisation from.

Example 2 Consider the following transfer function matrix (Gomez and Goodwin(2000))

$$
G(s)=\left[\begin{array}{cc}
1 & 0 \\
s+1 & \frac{s-2}{(s+1)^{2}}
\end{array}\right] .
$$

$G(s)$ is already in lower triangular form. The upper triangular form can be calculated as follows:

$$
\begin{aligned}
P_{u p}(s) & =G(s) U_{u p}(s) \\
& =\left[\begin{array}{cc}
1 & 0 \\
\frac{s+1}{s-1} & \frac{s-2}{(s+1)^{2}}
\end{array}\right]\left[\begin{array}{cc}
\frac{-(s-1)(s-2)}{(s+1)^{3}} & 1 \\
1 & 0
\end{array}\right] \\
& =\left[\begin{array}{cc}
\frac{-(s-1)(s-2)}{(s+1)^{3}} & 1 \\
0 & \frac{s+1}{(s-1)}
\end{array}\right] .
\end{aligned}
$$


Gomez and Goodwin (Gomez and Goodwin(2000)) shows that the lower triangular decoupling problem is not solvable for this system, and upper triangular decoupling is implementable based on stable coprime factorisation criteria proposed in (Gomez and Goodwin(2000)).

By checking TDD condition, we can easily obtain the same conclusion:

a) For lower triangular case:

We have $\delta\left[\tilde{G}_{+}(s)\right]=0 \leq \delta\left[G_{+}(s)\right]=1$. This shows $G(s)$ is not TDD. Thus, the lower triangular decoupling problem is unsolvable.

b) For upper triangular case:

We have $\delta\left[\tilde{P}_{+}(s)\right]=\delta\left[P_{+}(s)\right]=1$. This shows $P(s)$ is TDD. Thus, the upper triangular decoupling problem is solvable.

Now, we apply MFDs to test the solvability of triangular decoupling:

a) For low triangular case:

$$
\begin{aligned}
G(s) & =N(s) D^{-1}(s) \\
& =\left[\begin{array}{cc}
s-1 & 0 \\
s+1 & s-2
\end{array}\right]\left[\begin{array}{cc}
s-1 & 0 \\
0 & (s+1)^{2}
\end{array}\right]^{-1} .
\end{aligned}
$$

Because the first diagonal entries of $N(s)$ and $D(s)$ have common non-minimum phase zero 1, the lower triangular decoupling problem is unsolvable.

b) For upper triangular case:

$$
\begin{aligned}
& P_{u p}(s)=N_{u p}(s) D_{u p}^{-1}(s) \\
& =\left[\begin{array}{cc}
-(s-1)(s-2) & s-1 \\
0 & s+1
\end{array}\right]\left[\begin{array}{cc}
(s+1)^{3} & 0 \\
0 & s-1
\end{array}\right]^{-1} .
\end{aligned}
$$

Because the diagonal entries of $N_{u p}(s)$ and $D_{u p}(s)$ have no common nonminimum phase zero, the upper triangular decoupling problem is solvable.

It is also interesting to see that

$$
\left(\delta\left[|G(s)|_{+}\right]=0\right)<\left(\delta\left[G_{+}(s)\right]=1\right) .
$$

Just based on equation (3.2), Lemma 3 cannot draw any conclusion about the solvability of upper or lower decoupling problem for $G(s)$.

Remark 2 For decoupling or partial decoupling control, the first step is pairing. We have shown that the lower triangular decoupling problem of example 2 is not solvable. However, if we simply change the pairing, then the lower triangular decoupling problem is implementable.

After re-paring (redefine $u_{1}$ and $u_{2}$ as $\bar{u}_{2}$ and $\bar{u}_{1}$ ), $G(s)$ in example 2 can be written as follows:

$$
\bar{G}(s)=\left[\begin{array}{cc}
\frac{s+1}{s-1} & \frac{s-2}{(s+1)^{2}} \\
1 & 0
\end{array}\right] .
$$

The lower triangular form can be calculated as follows:

$$
\begin{aligned}
\bar{P}(s) & =\bar{G}(s) U(s) \\
& =\left[\begin{array}{cc}
\frac{s+1}{s-1} & \frac{s-2}{(s+1)^{2}} \\
1 & 0
\end{array}\right]\left[\begin{array}{cc}
1 & -\frac{(s-1)(s-2)}{(s+1)^{3}} \\
0 & 1
\end{array}\right] \\
& =\left[\begin{array}{cc}
\frac{s+1}{(s-1)} & 0 \\
1 & \frac{-(s-1)(s-2)}{(s+1)^{3}}
\end{array}\right] .
\end{aligned}
$$


$A s \delta\left\{\bar{P}_{+}(s)\right\}=\delta\left\{\bar{P}_{+}^{\tilde{(}}(s)\right\}=1, P(s)$ is $T D D$.

Furthermore,

$$
\begin{aligned}
\bar{P}(s) & =\left[\begin{array}{cc}
s+1 & 0 \\
s-1 & -(s-1)(s-2)
\end{array}\right]\left[\begin{array}{cc}
s-1 & 0 \\
0 & (s+1)^{3}
\end{array}\right]^{-1} \\
& =\left[\begin{array}{cc}
1 & 0 \\
\frac{s-1}{s+1} & \frac{-(s-1)(s-2)}{(s+1)^{3}}
\end{array}\right]\left[\begin{array}{cc}
\frac{s-1}{s+1} & 0 \\
0 & 1
\end{array}\right]^{-1}
\end{aligned}
$$

By checking the stable coprime factorisation condition and MFDs descried condition, we can see that after re-paring lower triangular decoupling is implementable.

\section{Conclusion}

This paper proved the TDD condition (which was regarded as restrictive in (Gomez and Goodwin(2000))) is actually equivalent with the algebraic condition (Gomez and Goodwin(2000)) for the triangular decoupling problem. A MFDs described necessary and sufficient condition is also provided. Based on these criteria, we can simplify the assessment of the solvability of the triangular decoupling problem.

\section{REFERENCES}

B.D.O. Anderson and N.T. Hung. Multivariable design reduction to scalar design problems. in Proc. Alternatives far Linear Multivariable Control, Nat. Eng. Consortium, Inc, Chicago, 88-95, 1978.

G.I. Gomez and G.C. Goodwin. An algebraic approach to decoupling in linear multivariable systems. International Journal of Control, 73:582-599, 2000.

N.T. Hung and B.D.O. Anderson. Triangularization technique for the design of multivariable control systems. IEEE Transactions on Automatic Control, 24:455-460, 1979.

N.T. Hung and B.D.O. Anderson. New multivariable frequency-domain-design techniques. Conference on Control Engineering, Melbourne, 144-148, 1979.

T. Kailath. Linear systems. Prentice-Hall Inc, New Jersey, 1980.

F.N. Kouvaritakis. Input-output triangular decoupling and data sensitivity. $A u$ tomatica, 32:569-573, 1996.

F.N. Koumboulis and M.G. Skarpetis. Robust triangular decoupling with application to 4ws cars. IEEE Transactions on Automatic Control, 45:344-352, 2000 .

K. Sugimoto and Y. Yamamoto. A polynomial matrix method for computing stable rational doubly coprime factorizations. Systems and Control Letters, 267-273, 1990.

A.I.G. Vardulakis. Linear Multivariable Control: Algebraic Analysis and Synthesis Methods. Wiley, Chichester, 1991.

M. Vidyasagar. Control system synthesis: a factorisation approach. The MIT Press, London, 1985. 


\section{A Proof of Lemma 2}

Proof: Intuitively, as $P(s)$ is proper, it have no poles at infinity. Because the zeros at infinity of each diagonal entry of $\bar{L}_{D_{p}}$ (over $\mathcal{S}$ ) are the poles of $P(s)$, we can draw the conclusion that there is no infinity zero in each diagonal entry of $\bar{L}_{D_{p}}$.

Now, we give a more explicit proof.

We can always find a description

$$
P(s)=N_{L}(s) \Lambda^{-1}(s),
$$

such that $N_{L}(s) \in \mathcal{R}[s]^{m \times m}$ is a lower triangular matrix, and $\Lambda(s) \in \mathcal{R}[s]^{m \times m}$ is a diagonal matrix. It should be note that the matrices $N_{L}(s)$ and $\Lambda(s)$ may not be right coprime.

Let $k_{j}, j \in\{1, \cdots m\}$ be the $j_{t h}$ column degrees of $\Lambda(s)$. Let

$$
d_{i}(s)=s^{k_{i}}+a_{i, k_{i}-1} s^{k_{i}-1}+\cdots+a_{i, 1} s+a_{i, 0} \in \mathcal{R} \quad i \in\{1, \cdots m\}
$$

be arbitrary polynomials with no zeros in closed right half plane $\mathcal{C}_{+}$and define

$$
\begin{aligned}
& D_{d}(s)=\operatorname{diag}\left[d_{1}(s), \cdots, d_{m}(s)\right] \in \mathcal{R}[s]^{m \times m}, \\
& A(s)=N_{L}(s) D_{d}(s)^{-1} \in \mathcal{S}^{m \times m}, \\
& B(s)=\Lambda(s) D_{d}(s)^{-1} \in \mathcal{S}^{m \times m} .
\end{aligned}
$$

Then, $P(s)=A(s) B^{-1}(s)$ with $A(s) \in \mathcal{S}^{m \times m}$ a proper rational lower triangular matrix and $B(s) \in \mathcal{S}^{m \times m}$ a proper rational triangular matrix. Furthermore, all diagonal entries of $B(s)$ are non-strictly proper.

As $P(s)=L_{N_{p}}(s) \bar{L}_{D_{p}}(s)=A(s) B^{-1}(s)$, we should be able to find a proper rational lower triangular matrix $C(s) \in \mathcal{S}^{m \times m}$ such that

$$
\begin{aligned}
& A(s)=L_{N_{p}}(s) C(s), \\
& B(s)=\bar{L}_{D_{p}}(s) C(s) .
\end{aligned}
$$

Now, we assume $\bar{L}_{D_{p}}(s)$ has an infinity zero in one of its diagonal entries. Then according to equation (A1), $C(s)$ should be non-proper as $B(s)$ is non-strictly proper. This is incorrect. Thus, $\bar{L}_{D_{p}}(s)$ should not have an infinity zero in one of its diagonal entries. 